\title{
Key individual identification using dimensional relevance in the stratum of networks
}

\author{
Article type: Research Article \\ Authors: Noor, Fozia (https://content.iospress.com:443/search?a=author\%3A\%28\%22Noor, Fozia\%22\%29). 마; * - I Akram, Muhammad Usman \\ (https://content.iospress.com:443/search?q=author\%3A\%28\%22Akram, Muhammad Usman\%22\%29). I Shah, Asadullah \\ (https://content.iospress.com:443/search?q=author\%3A\%28\%22Shah, Asadullah\%22\%29). | I Khan, Shoab Ahmad (https://content.iospress.com:443/search? \\ g=author\%3A\%28\%22Khan, Shoab Ahmad\%22\%29).
}

Affiliations: [a] Yanbu University College(YUC), Saudi Arabia | [b] International Islamic University Malaysia (IIUM), Malaysia | [c] National University of Sciences and Technology (NUST), Pakistan

Correspondence: [*] Corresponding author. Fozia Noor, Yanbu University College (YUC), Saudi Arabia. E-mail: fozia noor khan@yahoo.com (mailto:fozia_noor khan@yahoo.com).

Abstract: Different aspects of social networks have increasingly been under investigation from last decade. The social network studies range in various viewpoints from the structural and node measures to the information diffusion processes. Key node identification has been one of the limelight topics of social network analysis (SNA) specifically in a discipline like politics, criminology, marketing etc. This research uses multiple networks constructed from the different social site and real-life relationships to cover the multi-dimensional aspects of human relations. In the multi-relationship system, the different dimensions may differ in terms of relevance and weight. One of the most intriguing aspects of key node identification in the multi-dimensional system can be the consideration of dimension relevance. This research covers the methodology to optimize the weights of dimensions using a number of centrality measures from each network layer covering multiple different objectives of interest. The study formulates the novel weighted feature set pertaining to layer relevance calculated based on layers relative importance through particle swarm optimization technique. The framework applies ensemble-based approach on the weighted feature set along with node characteristics to predict key nodes in a network. The results are validated against ground truth data and accuracy achieved is promising.

Keywords: Multi-layer social networks, dimensional relevance, key player identification, majority voting based ensemble, particle swarm optimization

DOI: $10.3233 / J I F S-181517$

Journal: Journal of Intelligent \& Fuzzy_Systems (https://content.iospress.com:443/journals/journal-of-intelligent-and-fuzzy-systems), vol. 37, no. 2, pp. 2153-2167, 2019

Published: 09 September 2019

Price: EUR 27.50 
